\title{
Variability in the mating calls of the Lusitanian toadfish Halobatrachus didactylus: cues for potential individual recognition
}

\author{
M. C. P. Amorim* and R. O. Vasconcelos \\ Unidade de Investigação em Eco-Etologia. I.S.P.A. Rua Jardim do Tabaco 34, 1149-041 \\ Lisboa, Portugal
}

(Received 12 October 2007, Accepted 22 May 2008)

\begin{abstract}
The mating sounds (boatwhistles) of nesting batrachoidid Halobatrachus didactylus males were recorded in the Tagus Estuary from piers. Thirteen males with 16 boatwhistles per fish were analysed for 20 acoustic features. All variables showed larger between-male than within-male variation and differed significantly among individuals. Discriminant function analyses (DFA) considering seven of these variables assigned $90-100 \%$ of boatwhistles to the correct individual, depending on the number of males and number of sounds per male included in the model. The acoustic features that consistently best discriminated individuals were the dominant frequency of the middle tonal segment of the boatwhistle $\left(\mathrm{P}_{2}\right)$ and dominant frequency modulation, followed by $\mathrm{P}_{2}$ pulse period, amplitude modulation and sound duration. These results suggest the possibility of individual recognition based on acoustic cues.

(C) 2008 The Authors

Journal compilation (C) 2008 The Fisheries Society of the British Isles
\end{abstract}

Key words: acoustic communication; Batrachoididae; individuality; signal variability; sound production.

\section{INTRODUCTION}

The ability to discriminate between individuals or groups of individuals is important for the establishment of social relations and implies individual distinctiveness (Bradbury \& Vehrencamp, 1998). Individuality in acoustic signalling (vocal signatures) arises when the within-individual variation is smaller than the variation between individuals in one or more acoustic characteristics or when individuals differ in the presence or absence of particular vocal features (Beecher, 1989; Bee et al., 2001). Individual identification through vocal signatures can mediate kin recognition (Jouventin et al., 1999), territorial neighbour recognition (Bee \& Gerhardt, 2001), mate-pair recognition (Speirs \& Davis, 1991) and true individual recognition (Sayigh et al., 1999).

The existence of individual characteristics in vocal signals is well known in various groups of animals including mammals, birds and amphibians (Bee

*Author to whom correspondence should be addressed. Tel.: +351 218811700; fax: +351 218860954; email: amorim@ispa.pt 
et al., 2001; Christie et al., 2004) but has been poorly studied in fishes (Amorim, 2006). The most common intraspecific variation in fish sounds is an inverse relation of dominant frequency with fish size that may mediate individual recognition based on size information (Myrberg \& Riggio, 1985; Myrberg et al., 1993). More elaborate (multi-featured) individual differences in fish sounds occur in Batrachoididae (toadfishes; Barimo \& Fine, 1998; Edds-Walton et al., 2002; Thorson \& Fine, 2002a, b; Fine \& Thorson, 2008) and Mormyridae (weakly electric fishes; Crawford et al., 1997; Lamml \& Kramer, 2006). In both families, territorial males rely on their advertisement calls to attract females in turbid waters or at night (Winn, 1967; Crawford et al., 1997). Additionally, these calls are involved in male--male competition (Winn, 1967; Remage-Healy \& Bass, 2005). It has been suggested that mating calls may promote individual recognition in these animals. For example, differences in waveform, sound duration and distribution of energy in different harmonic bands allow clear identification of different male toadfishes [Opsanus tau (L., 1766) and Opsanus beta (Goode \& Bean, 1880)] recorded through passive acoustics (Edds-Walton et al., 2002; Thorson \& Fine, 2002a).

Despite the clear indication of the existence of complex acoustic signals in batrachoidids and mormyrids that may involve individual recognition, there is to date no detailed statistical analysis of individual variation in fish sounds. The goal of the present study is to describe in detail the boatwhistles of nesting Lusitanian toadfish Halobatrachus didactylus (Bloch \& Schneider) males and to determine which signal properties may potentially mediate individual recognition. A comparison of the intra- with inter-male variability in 20 acoustic features was made. Multivariate statistics were used to identify the best variables to discriminate between individuals. A preliminary study has shown that different nesting $H$. didactylus males can be recognized by ear and easily identified through inspection of the spectrogram and oscillogram of their mating sounds (Amorim et al., 2006). Moreover, this species has an unusual large acoustic repertoire for fishes, consisting of at least five distinct sound types (dos Santos et al., 2000; Amorim et al., 2008), suggesting it has a complex acoustic communication system. This study provides a basis for future playback experiments in order to test for individual recognition among nesting males and support the use of acoustic cues in mate attraction and choice.

\section{MATERIALS AND METHODS}

\section{STUDY SPECIES}

Halobatrachus didactylus is an eastern Atlantic member of the Batrachoididae that occurs in estuaries and coastal lagoons (Roux, 1986). During the reproductive season, that lasts in Portugal from May to July (Modesto \& Canário, 2003), breeding males defend nests under rocks in shallow water. Nesting males use an advertisement call (the boatwhistle) to attract females that results from the contraction of sonic muscles attached to the swimbladder (dos Santos et al., 2000). Spawning females attach their eggs to the roof of a nest and leave the area, while the resident males provide parental care until the young are free-swimming (Roux, 1986; dos Santos et al., 2000). As in other batrachoidids, a second type of male with different morphometric and endocrine characteristics is thought to use a sneaking strategy for mating (Brantley \& Bass, 1994). 
These type II males have larger testes, smaller sonic muscle mass and lower levels of 11-ketotesterone than nesting males (Modesto \& Canário, 2003).

Since territorial males nest close together, boatwhistles are emitted in choruses resulting in a very conspicuous acoustic output (dos Santos et al., 2000). In the peak of the breeding season, a small aggregation of males vocalizing close to the hydrophone can reach an average of 30 boatwhistles $\min ^{-1}$ (Amorim et al., 2006).

The boatwhistle is a tonal multi-harmonic sound lasting c. $800 \mathrm{~ms}$ (Amorim et al., 2006). The fundamental frequency is $c .60 \mathrm{~Hz}$ [H1; Fig. 1(b)] and the dominant frequency is typically either the second or the fourth harmonic (Fig. 1) (Amorim et al., 2006).

\section{RECORDING AND ACOUSTIC ANALYSIS OF BOATWHISTLES}

Several recording sessions lasting from 5 to $10 \mathrm{~min}$ were carried out during the mating season in July 2001 and July 2002 in two areas within the Tagus Estuary, Portugal: Montijo $\left(38^{\circ} 42^{\prime} \mathrm{N} ; 8^{\circ} 58^{\prime} \mathrm{W}\right)$ and Barreiro $\left(38^{\circ} 39^{\prime} \mathrm{N} ; 9^{\circ} 04^{\prime} \mathrm{W}\right)$. These areas had been previously identified as $H$. didactylus breeding areas (Amorim et al., 2006). Moreover, nesting males were also observed to call in nests exposed at low spring tides at these locations (pers. obs.). During recording periods, water temperature ranged between 21-22 C. A hydrophone [High Tech 94 SSQ (High Tech Inc., Gulfport, MS,

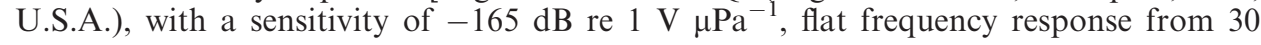
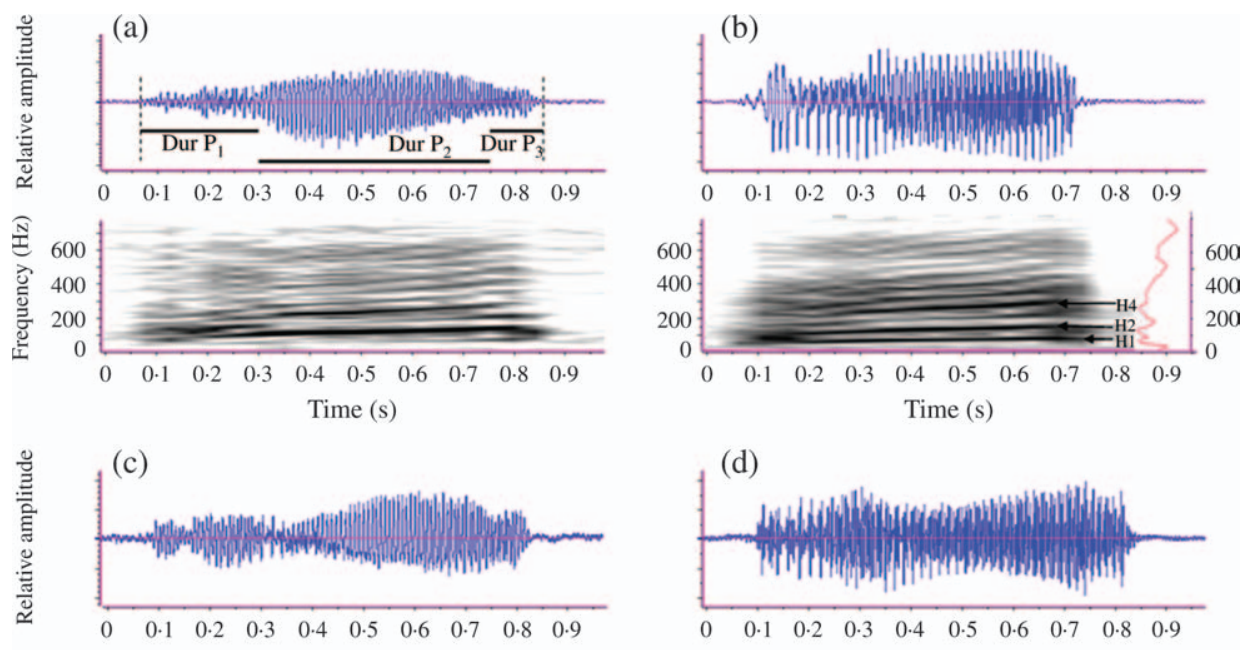

(d)
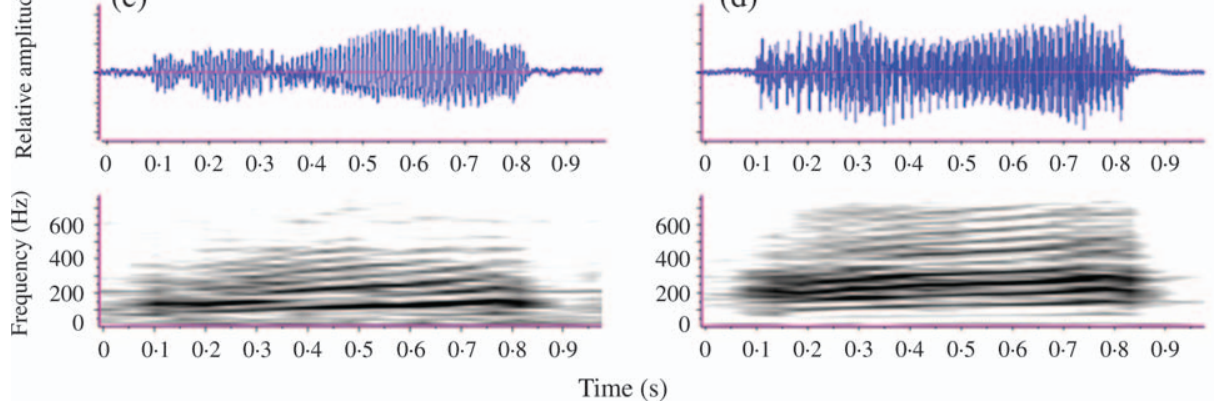

FIG. 1. (a), (b), (c) and (d) Oscillograms and sonograms of boatwhistles emitted by four nesting Halobatrachus didactylus males. The middle tonal phase of a boatwhistle $\left(\mathrm{P}_{2}\right)$ dominant frequency coincides with the second harmonic (H2) in (a), (b) and (c), and with the fourth harmonic (H4) in male (d), which are multiples of the fundamental frequency (H1). A power spectrum of phase 2 is given for male (b). Dur $\mathrm{P}_{1}$, Dur $\mathrm{P}_{2}$ and Dur $\mathrm{P}_{3}$ duration of phases 1, 2 and 3 of the boatwhistle. The dotted line depicts total boatwhistle duration. Sampling frequency 44 kHz; FFT size 8192 points; Hamming window. 
$\mathrm{Hz}$ to $6 \mathrm{kHz} \pm 1 \mathrm{~dB}$ ] was lowered from piers in these two locations in several sites where acoustic activity was evident. The hydrophone was $c .150-200 \mathrm{~mm}$ above the substratum. Recording sites within the same pier were at least $4 \mathrm{~m}$ apart and each recording session was made from a different site. Water depth varied approximately between 2 and $5 \mathrm{~m}$ depending on tide.

Sounds were recorded on tape (Sony TCD-D8, $44 \mathrm{kHz}, 16$ bit resolution; Sony, Tokyo, Japan) and the analogue output of the recorder was digitized with a similar sampling frequency and resolution to a computer with a sound capture device (Edirol UA-5; Roland, Osaka, Japan). Sound files were analysed with Raven 1.2.1 for Windows (Bioacoustics Research Program, Cornell Laboratory of Ornithology, Cornell, NY, U.S.A.). A total of seven recording sessions were considered. Sound analysis was restricted to the males calling close to the hydrophone that presented a high signal to noise ratio $(\mathrm{SNR})$ (mean $=29 \mathrm{~dB}$; the minimum SNR considered was of $18 \mathrm{~dB}$ ) and a maximum of three individuals was considered per recording session. Only one recording session was considered per male. Distinction of different individuals between years was assured by considering males from different location, i.e. Montijo in 2001 $(n=7)$ and from Barreiro in $2002(n=6)$. Distinction of different males in the same recording session was based on differences in waveform envelope and relative sound amplitude that reflected the distance of the calling male to the hydrophone (an example is depicted in Fig. 2). As expected, relative sound amplitude of a particular male remained constant throughout a recording session since nesting males are stationary for long periods (dos Santos et al., 2000), especially in the peak of the breeding season when territories are already established and males call to attract females while caring for their young (Barimo \& Fine, 1998; Knapp et al., 1999). To ensure that the distance between recording sites (minimum of $4 \mathrm{~m}$ ) sufficed to prevent considering a male twice, boatwhistles were played back at the recording locations and recorded simultaneously at different distances from the speaker with similar gains. The playback audio chain consisted of a laptop computer, an amplifier (Phoenix Gold QX 4040, Portland, OR, U.S.A.) and a speaker (Electrovoice UW-30; Lubell Labs Inc. Columbus, OH, U.S.A.) placed $150 \mathrm{~mm}$ above the substratum. Played-back sounds were recorded with a second laptop computer, a sound capture device (Edirol UA25; Roland) and three hydrophones (High Tech $94 \mathrm{SSQ}$ ) placed $c .150-200 \mathrm{~mm}$ above the substratum and at $0.5,1.5$ and $4 \mathrm{~m}$ from the speaker. Amplitude of sound playback was determined by recording a male in a closed nest with the same recording settings as during recordings of sound playback. The nest was naturally occupied by the male and its entrance was closed with a plastic mesh that allowed prey items to enter the nest but prevented the subject male from abandoning the nest during recordings. The acoustic energy fell off very rapidly ( $21 \mathrm{~dB}$ loss from $0.5 \mathrm{~m}$ to $4 \mathrm{~m}$ from the speaker) and at $4 \mathrm{~m}$ away from the speaker, the boatwhistle could hardly be distinguished from the background noise (Fig. 3). The marked sound attenuation observed in the study sites, typical of shallow waters (Fine \& Lenhardt, 1983), strongly suggests that the males considered

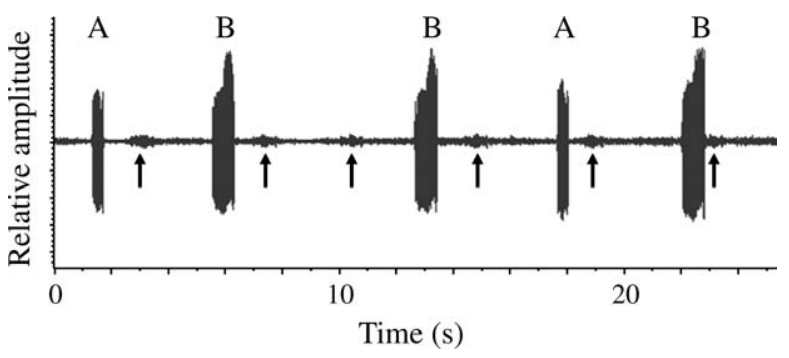

FIG. 2. Example of boatwhistles produced by two male Halobatrachus didactylus (A and B) that can be distinguished by the waveform envelope and relative sound amplitude, i.e. distance from the hydrophone. Arrows point at background boatwhistles. 


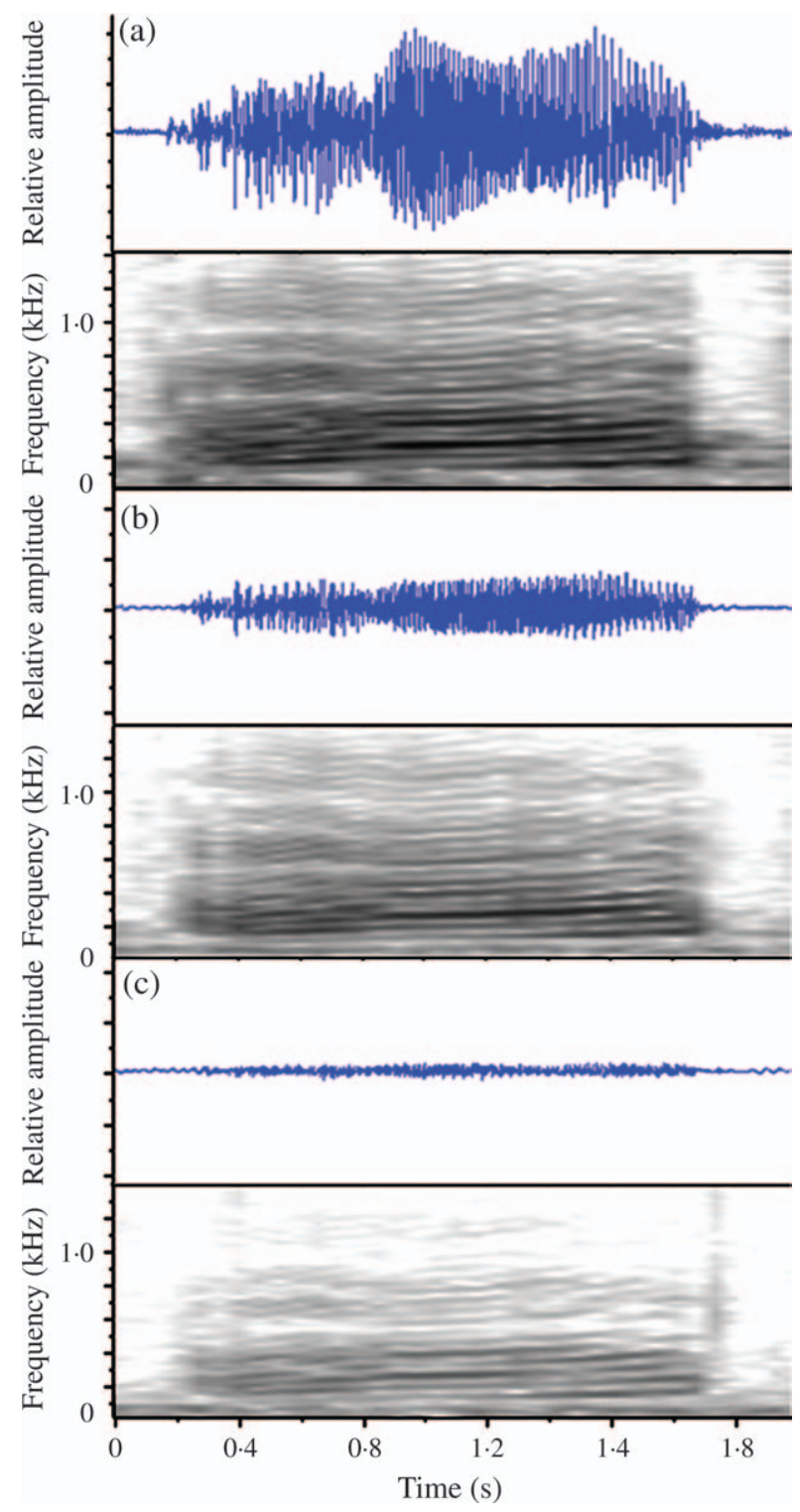

FIG. 3. Oscillograms and sonograms of a Halobatrachus didactylus boatwhistle played back by an underwater speaker recorded at (a) $0 \cdot 5$, (b) 1.5 and (c) $4 \mathrm{~m}$ away from the source. The acoustic energy of boatwhistles suffered an average attenuation of $21 \mathrm{~dB}$ from 0.5 to $4 \mathrm{~m}$ from the speaker and at $4 \mathrm{~m}$ could hardly be distinguished from the background noise. Sampling frequency $44 \mathrm{kHz}$; fast fourier transform (FFT) size 8192 points; Hamming window.

in the present study are distinct individuals. Other studies have used similar criteria to the present study to identify unseen toadfishes in sound recordings (Edds-Walton et al., 2002; Thorson \& Fine, 2002a, b) and, in one occasion, male identity was confirmed through diving (Barimo \& Fine, 1998).

A total of 13 males with 16 boatwhistles per fish was analysed for 20 acoustic features. The classification used by dos Santos et al. (2000) that considers three distinct 
phases in the boatwhistle [beginning $\left(\mathrm{P}_{1}\right)$, middle $\left(\mathrm{P}_{2}\right)$ and end $\left(\mathrm{P}_{3}\right)$ ] was adopted. These three phases differ in pulse period and dominant frequency (dos Santos et al., 2000), with the pulse period typically decreasing and the dominant frequency increasing from phases 1-3 (see Table I). The identification of these phases was also based on differences in sound amplitude (Fig. 1) and fine waveform structure (Fig. 4). The following acoustic variables were measured: sound duration $(\mathrm{ms})$, measured from the start of the first pulse (when acoustic energy appears above the background noise) to the end of the last pulse [Fig. 1 (a)]; duration of the segments $\mathrm{P}_{1}, \mathrm{P}_{2}$ and of $\mathrm{P}_{3}$ (ms) [Fig. 1 (a)]. Relative $\mathrm{P}_{2}$ duration was calculated by dividing the duration of $\mathrm{P}_{2}$ by the total sound duration and was expressed as a percentage; pulse period in $\mathrm{P}_{1}, \mathrm{P}_{2}$ and $\mathrm{P}_{3}(\mathrm{~ms})$, calculated as the average peak-to-peak interval between six consecutive pulse units in the middle of each segment, except in $\mathrm{P}_{3}$ that considered the whole segment (Fig. 4); number of pulses in $\mathrm{P}_{1}, \mathrm{P}_{2}, \mathrm{P}_{3}$ and the total number of pulses in the whole sound; dominant frequency $(\mathrm{Hz})$, i.e. the frequency with maximum energy, was determined in $\mathrm{P}_{1}$, $\mathrm{P}_{2}, \mathrm{P}_{3}$ and in the entire sound. Fundamental frequency was calculated as the inverse of the average pulse period measured in $\mathrm{P}_{1}$ and $\mathrm{P}_{2}$. In batrachoidids, the fundamental frequency of the mating signals is determined by the rate of contraction of the sonic muscles attached to the swimbladder (Skoglund, 1961; Fine et al., 2001). These measurements were confirmed with the power spectra [Fig. 1(b)] and were preferred to measuring the fundamental frequency directly because in many fish this frequency band had little energy. Dominant frequency modulation was calculated by dividing $\mathrm{P}_{1}$ by $\mathrm{P}_{2}$ dominant frequencies and fundamental frequency modulation was calculated in a similar way; amplitude modulation was similarly calculated by dividing the mean amplitude (RMS) measured for the $\mathrm{P}_{1}$ segment by the one measured for the $\mathrm{P}_{2}$ segment; RMS amplitude is a measurement native to Raven software. Time to maximum amplitude was measured from the start of the first pulse to the sound peak amplitude; this is also a measurement native to Raven software.

Temporal variables were measured from oscillograms and the dominant frequencies from power spectra [fast fourier transform (FFT) size 8192 points; Hamming window].

\section{STATISTICAL ANALYSIS}

Mean \pm S.D. values were calculated for the above 20 acoustic features for all males. Overall means, s.D. and range values were subsequently calculated using each male mean values for each variable. In order to compare between-male with within-male variability for each acoustic feature the within-male coefficient of variance (C.v.w $=$ S.D.:mean) was calculated and compared with the between-male coefficient of variation (C.V.b). The C.V.b was obtained by dividing the overall S.D. by the respective overall mean. The ratio C.V.b:C.V.w was calculated to obtain a measure of relative between-male variability for each boatwhistle feature. When this ratio assumes values larger than one, it suggests that an acoustic feature could be used as a cue for individual recognition (Bee et al., 2001; Christie et al., 2004). Kruskal-Wallis analysis was used to test for differences between males for each acoustic variable. Non-parametric statistics were preferred to parametric ANOVAs due to the lack of homoscedasticity of variances.

Discriminant function analysis (DFA) was carried out using SPSS 15.0 for Windows (SPSS Inc., Chicago, IL, U.S.A.) as a multivariate tool to determine which acoustic features best discriminate between males. DFA also gives a measure of discrimination accuracy by revealing the percentage of sounds assigned to the correct individual. Only seven of the 20 acoustic variable were considered for the DFA: total sound duration, relative $\mathrm{P}_{2}$ duration, $\mathrm{P}_{2}$ pulse period, $\mathrm{P}_{2}$ dominant frequency, dominant frequency modulation, fundamental frequency modulation and amplitude modulation. These variables were chosen because they were uncorrelated, had a C.V.b:C.V.w ratio larger than one and presented a low C.V.w $(\leq 0 \cdot 1$; Table I). To assess the predictive accuracy of the models obtained, a cross-validation method ('leave-one-out') was carried out. In this method each sound is classified by the discriminant functions derived by the $n-1$ remaining sounds. Because the $\mathrm{H}$. didactylus emits boatwhistles in aggregations of different sizes, further DFA were performed to explore the variation of classification success with fish 


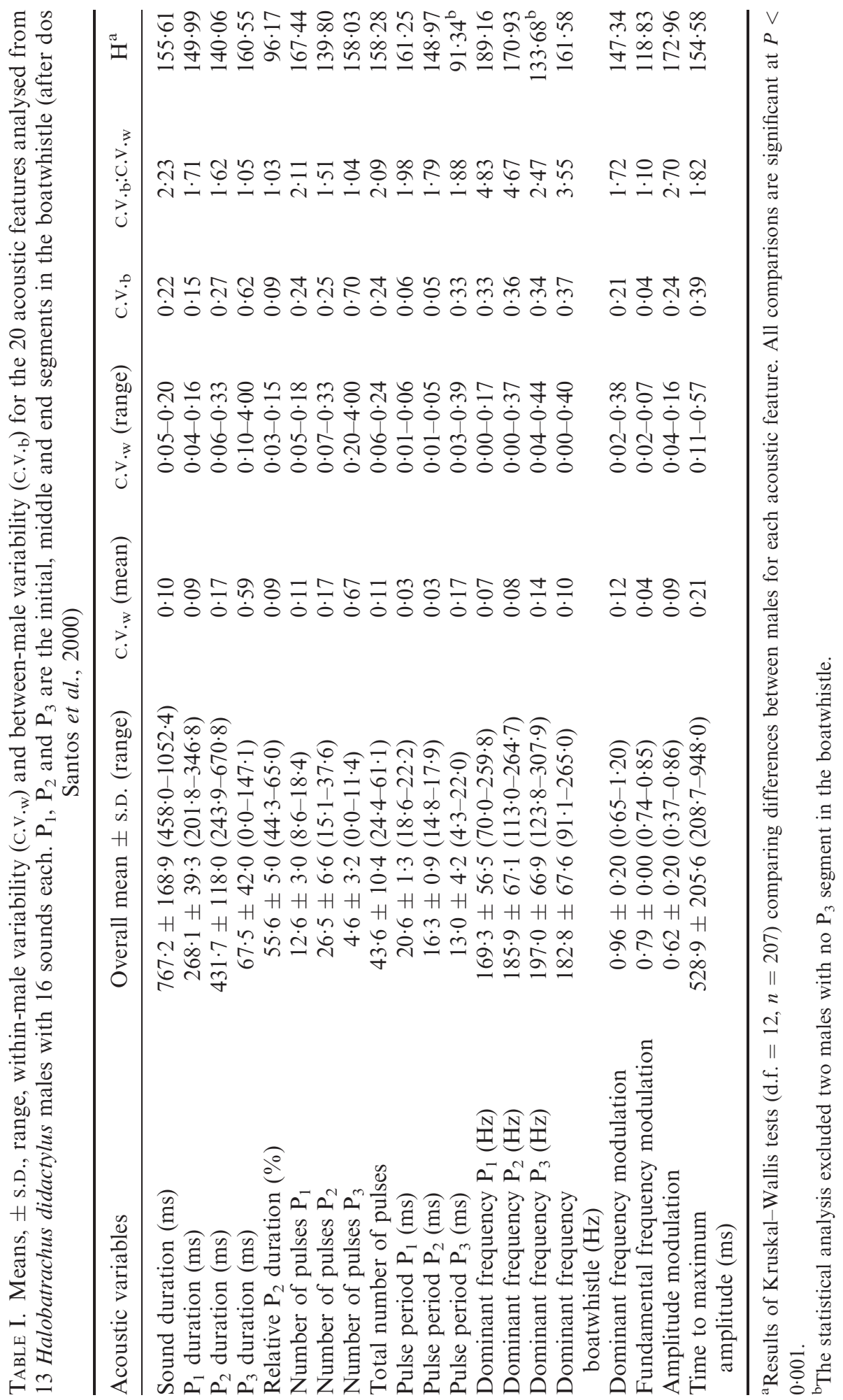




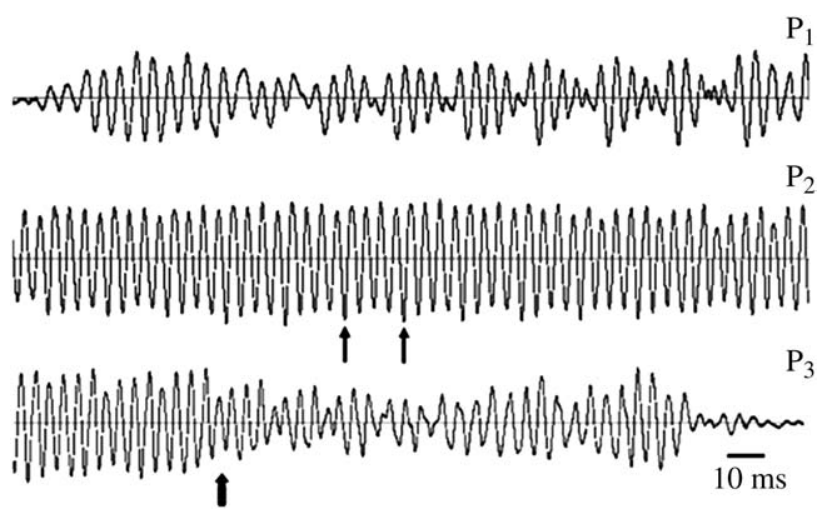

FIG. 4. Oscillograms of the initial $\left(\mathrm{P}_{1}\right)$, the middle tonal place $\left(\mathrm{P}_{2}\right)$ and the end phase $\left(\mathrm{P}_{3}\right)$ of a Halobatrachus didactylus boatwhistle. Thin arrows indicate the peak amplitude of two consecutive pulses, i.e. the pulse period. The thick arrow depicts the start of $\mathrm{P}_{3}$. Note the differences in the fine waveform structure among the boatwhistle phases.

group size. Ten groups of males were considered. Each group consisted of randomly chosen males from the initial data set in various sample sizes: three, five, eight and 11 males. Five and 10 boatwhistles randomly chosen per male for each male group were used to further verify the change in classification accuracy with the number of sounds considered in the analysis.

\section{RESULTS}

\section{BOATWHISTLE STRUCTURE}

The mating sounds of the $H$. didactylus varied considerably in duration ranging from 317 to $1290 \mathrm{~ms}(n=207$ sounds analysed from all males), with average values of $767 \mathrm{~ms}$ (Table I). The fundamental frequency (H1) and the harmonics (multiples of $\mathrm{H} 1$ ) showed a slight frequency modulation that was more obvious in the higher harmonics (Fig. 1). H1 was the dominant frequency in only one male that exhibited eight of the 16 sounds analysed with dominant frequencies in the $\mathrm{H} 1$ and the remaining in the $\mathrm{H} 2$ (see male 5 in Fig. 5). H4 was the most common dominant frequency $(51 \cdot 2 \%)$ followed by H2 (44.9\%).

The three segments $\left(\mathrm{P}_{1}, \mathrm{P}_{2}\right.$ and $\left.\mathrm{P}_{3}\right)$ that make up the boatwhistle (dos Santos et al., 2000) were characterized by different durations, pulse periods, relative amplitude and dominant frequencies (Table I). The tonal phase of the boatwhistle $\left(\mathrm{P}_{2}\right)$ was the longest segment, lasting on average $56 \%$ of the sound, and exhibited an intermediate pulse period and dominant frequency to $\mathrm{P}_{1}$ and $\mathrm{P}_{3}$. The boatwhistle dominant frequency typically corresponded to $\mathrm{P}_{2}$ dominant frequency. Pulses in $\mathrm{P}_{1}$ and $\mathrm{P}_{3}$ were of a more irregular shape and had clear starts and ends (Fig. 4), while pulses in $\mathrm{P}_{2}$, the tonal segment, were more regular and fused together sometimes resembling a sinusoidal wave (Fig. 4). The third boatwhistle segment was more variable in duration, pulse number, pulse period and dominant frequency than the two previous segments (Fig. 4 and Table I) and was not present in all males. Two males never exhibited 


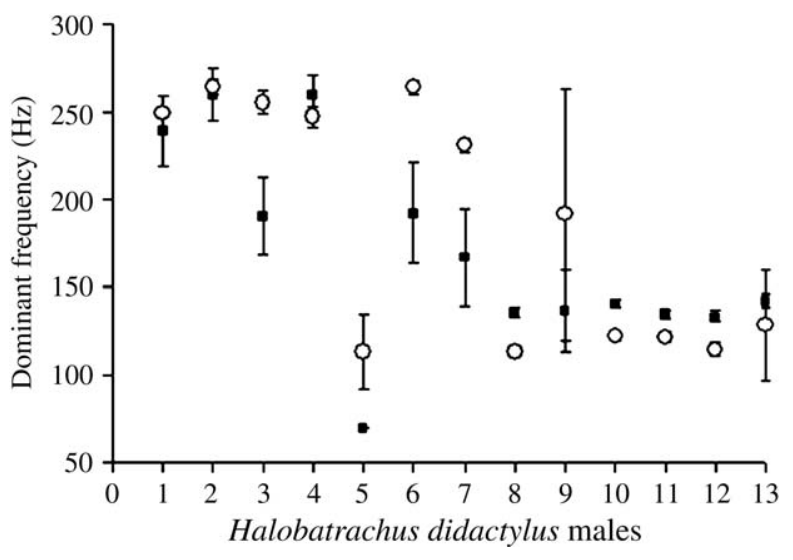

FIG. 5. Mean \pm S.D. dominant frequencies of Halobatrachus didactylus boatwhistle segments initial phase $\left(\mathrm{P}_{1}\right)$

and middle tonal phase $\left(\mathrm{P}_{2}\right)(\mathrm{O})$ in the 13 males analysed.

the segment $\mathrm{P}_{3}$ in their boatwhistles and in another male it was present only in some of the calls analysed.

\section{INDIVIDUALITY}

Boatwhistles were distinct between individuals in terms of waveform (amplitude modulation) and spectral characteristics (Fig. 1). Detailed waveform patterns were also distinctive among calling males (Fig. 6). There was a strong stereotypy in most acoustic variables measured, with half of these features showing within-male C.V.s $\leq 0 \cdot 10$ (Table I). All the 20 features analysed had C.V.b:C.V.w ratios $>1$, showing that they were more variable among than within males. Consistently, the Kruskal-Wallis analyses demonstrated significant differences among males for all features (Table I), indicating that these acoustic variables can potentially provide recognition cues to identify calling males. The larger relative between-male variability (larger $\mathrm{C} \cdot \mathrm{V} \cdot \mathrm{b}: \mathrm{C} \cdot \mathrm{V} \cdot \mathrm{w}$ ratios) corresponded to the dominant frequencies of $\mathrm{P}_{1}$ and $\mathrm{P}_{2}$ and of the whole signal (Table I and Fig. 5). Most males presented dominant frequencies of $\mathrm{P}_{2}$ and of the whole boatwhistle either in the $\mathrm{H} 2$ or in the $\mathrm{H} 4$ and showed little within-male variation (Fig. 5). Three males exhibited, however, higher withinmale variability in this feature (males 5, 9 and 13 in Fig. 5) because the dominant frequency in different sounds corresponded to different harmonic bands. Figure 6 also illustrates that approximately half of the males had lower dominant frequencies in $\mathrm{P}_{1}$ than in $\mathrm{P}_{2}$, whereas the remaining males showed an opposite trend.

A discriminant function analysis using only seven uncorrelated acoustic features generated a significant model (DFA, $n=207$, d.f. $=84,1159, P<0 \cdot 001$ ). The three first discriminant functions explained almost all data variability (91\%; Table II). The sound features which weighted most heavily in explaining variation in the first three discriminant functions were $\mathrm{P}_{2}$ dominant frequency followed by dominant frequency modulation for the first function, $\mathrm{P}_{2}$ pulse period followed by amplitude modulation for the second function and total 
(a)

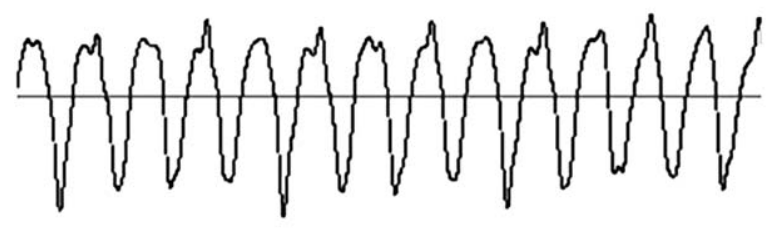

(b)

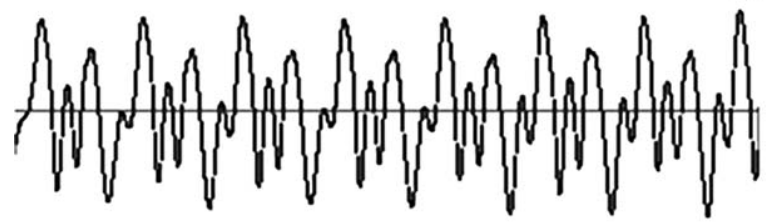

(c)

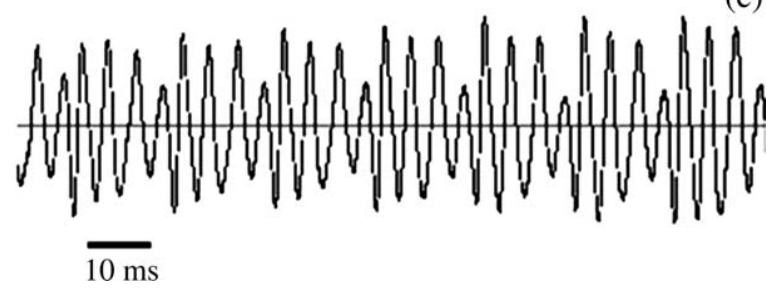

Fig. 6. (a), (b) and (c) Boatwhistles from different Halobatrachus didactylus males show differences in the waveform details in the tonal phase $\left(\mathrm{P}_{2}\right)$.

duration for the third function (Table II). The highest correlations between the discriminant variables and these discriminant functions were $\mathrm{P}_{2}$ dominant frequency for the first, amplitude modulation for the second and total sound duration for the third discriminant functions. Classification success averaged $90 \cdot 9 \%$ [ \pm S.D. (range) $= \pm 10 \cdot 4 \%(68 \cdot 8-100 \%)]$ and was significantly greater than the classification expected by chance ( $a$ priori probability range $=0.072$ 0.077; Wilcoxon test, $n=13, P<0 \cdot 01)$. A clear separation between individuals in the two-dimensional space defined by the first two discriminant functions is depicted in Fig. 7. After cross-validation, the correct classification decreased to a mean \pm S.D. of $85 \cdot 6 \pm 16 \cdot 8 \%$ with values ranging from $56 \cdot 3-100 \%$.

Subsequent discriminant analyses, including the same seven acoustic features, explored variation of classification success with fish group size (three, five, eight and 11 males) and number of sounds (five and 10) per male. The mean percentage of correct classification increased in groups of fewer males from c. $90 \%$ (11 males) to $100 \%$ (three males) of boatwhistles assigned to the correct individual (Fig. 8). There was no difference in the classification success between the analyses that included 10 boatwhistles per male and those that included only five boatwhistles, except in the sample size of eight males where mean correct classification values were 3.5\% higher in the five boatwhistle analysis (Fig. 8; 95\% CI). Classification success was thus consistently high even when considering few calls per individual in relatively large groups. For example, the analysis that included 10 random groups of 11 males with five randomly assigned 
TABLE II. Standardized canonical discriminant function analysis (DFA) coefficients, eigenvalues and cumulative percentage of variance explained by the first three discriminant functions of a DFA classifying Halobatrachus didactylus males $(n=13)$ by their boatwhistle $(n=16)$ characteristics

\begin{tabular}{lccc}
\hline & \multicolumn{3}{c}{ Discriminant functions } \\
\cline { 2 - 4 } Discriminant variables & First & Second & Third \\
\hline Sound duration & $0 \cdot 34$ & $0 \cdot 35$ & $-1 \cdot 22^{\mathrm{a}}$ \\
Relative $\mathrm{P}_{2}$ duration $(\%)$ & $-0 \cdot 39$ & $0 \cdot 04$ & $0 \cdot 78$ \\
$\mathrm{P}_{2}$ pulse period & $-0 \cdot 44$ & $0 \cdot 83$ & $-0 \cdot 04$ \\
$\mathrm{P}_{2}$ dominant frequency & $1 \cdot 55^{\mathrm{a}}$ & $0 \cdot 30$ & $0 \cdot 56$ \\
Dominant frequency modulation & $1 \cdot 26$ & $0 \cdot 57$ & $0 \cdot 58$ \\
Fundamental frequency modulation & $0 \cdot 26$ & $-0 \cdot 60$ & $0 \cdot 15$ \\
Amplitude modulation & $0 \cdot 05$ & $-0 \cdot 77^{\mathrm{a}}$ & $-0 \cdot 41$ \\
Eigenvalue & $22 \cdot 68$ & $11 \cdot 56$ & $6 \cdot 10$ \\
Cumulative \% of variance & $51 \cdot 1$ & $77 \cdot 1$ & $90 \cdot 8$ \\
\hline
\end{tabular}

$\mathrm{P}_{2}$, middle tonal segment of the boatwhistle.

${ }^{a}$ Discriminant variable with the highest pooled within-groups correlations with the standardized discriminant functions.

sounds, revealed a mean correct classification of $92.5 \%$, which is well above the classification expected by chance alone. As with the initial DFA, which considered the whole data set, the acoustic features that loaded more heavily in the first two discriminant functions of these analyses were $\mathrm{P}_{2}$ dominant frequency and dominant frequency modulation (typically in the first discriminant function) as well as $\mathrm{P}_{2}$ pulse period, amplitude modulation and sound duration.

Predictive accuracy of the above models (calculated by the cross-validation leave-one-out procedure) considering different group sizes of randomly selected

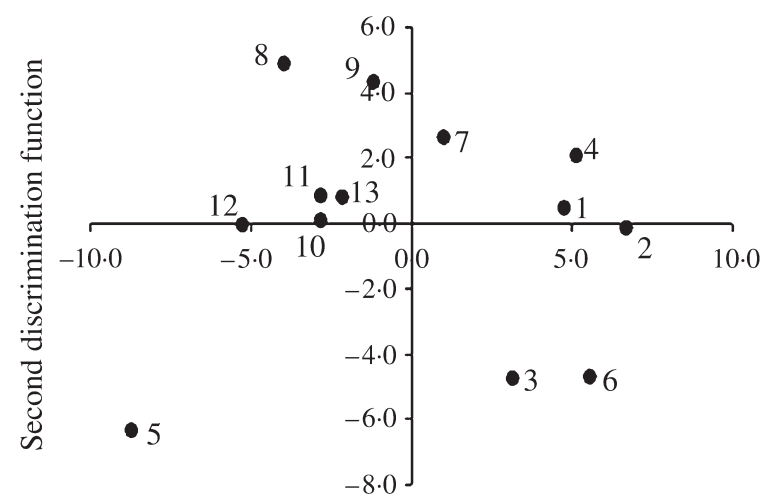

First discriminant function

FIG. 7. Representation of the 13 Halobatrachus didactylus males (boatwhistle group centroids) in the bidimensional space defined by the first two discriminant functions of a discriminant function analysis considering seven acoustic features. Middle tonal phase $\left(\mathrm{P}_{2}\right)$ dominant frequency correlates with the first discriminant function and amplitude modulation with the second. 


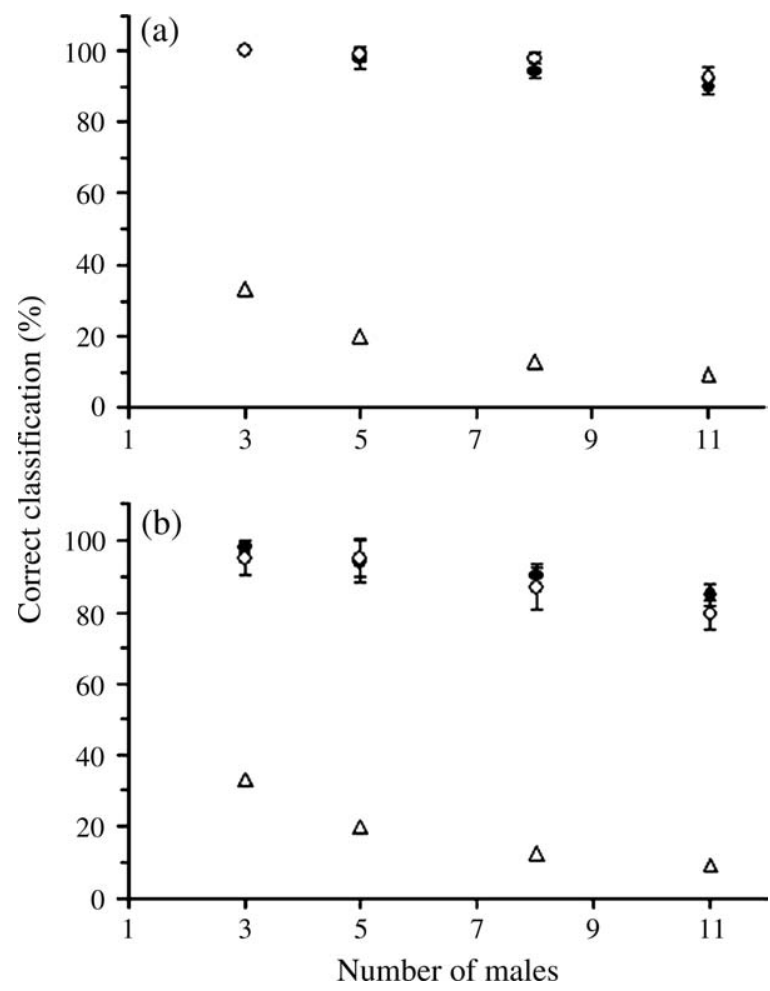

FIG. 8. (a) Variation of mean $\pm 95 \%$ CI classification success with fish group size (10 groups of three, five, eight and 11 randomly chosen Halobatrachus didactylus males) and number of sounds per individual: 5 $(\bigcirc)$ and 10 (O) boatwhistles. (b) Similar percentages of classification success obtained after crossvalidation $(\triangle$, the classification success expected from randomly assigning calls to the different male).

males also yielded high estimates of correct classifications. When 10 sounds were considered per male, the percentage of correct classification varied from an average of $98-85 \%$ in groups of three to 11 males, respectively (Fig. 8). Similar results were obtained when considering five boatwhistles per male with classification success decreasing from $95 \%$ in groups of three males to $79 \%$ in groups of 11 males (Fig. 8). In conclusion, after cross-validation these analyses still assigned high percentages of sounds to the correct males and considerably more than expected by chance.

\section{DISCUSSION}

The boatwhistles emitted by the $H$. didactylus consisted of a relatively long series of rapidly repeated pulses with average duration around $770 \mathrm{~ms}$. These sounds exhibited the fundamental frequencies at $c .60 \mathrm{~Hz}$ with typical dominant frequencies represented by the second or the fourth harmonic bands. The boatwhistle of $H$. didactylus was very similar to the one of $O$. tau, which starts with a wide-frequency non-harmonic grunt-like phase caused by slower and more irregular sonic muscle contractions, followed by a longer tonal 
segment (Fine, 1978). Boatwhistles of the latter species are, however, shorter (200-500 ms) and have higher fundamental frequencies (c. $200 \mathrm{~Hz}$ ) than the boatwhistles of the H. didactylus (Fine, 1978; Barimo \& Fine, 1998; Edds-Walton et al., 2002). Other well-studied batrachoidids produce more divergent calls. Opsanus beta emits a more complex courtship sound with fundamental frequencies around $270-280 \mathrm{~Hz}$ that starts with zero to three grunts followed by a long tonal ('boop') note and up to three shorter boops lasting over a second (Thorson \& Fine, 2002a). Nesting Porichthys notatus Girard, males emit remarkably long courtship sounds ('hums') that last from seconds to over an hour, with fundamental frequencies around $100 \mathrm{~Hz}$ (Ibara et al., 1983; Brantley \& Bass, 1994).

Clear differences were found among boatwhistles attributed to different males that can potentially be used in individual recognition. All variables were significantly more variable between than within males and thus could all potentially provide cues to identify individuals. A DFA using a sub-set of the initial acoustic features assigned boatwhistles to the correct male in $91 \%$ of cases, and in $86 \%$ of cases after cross-validation, showing a high predictive accuracy. Classification success of boatwhistles varied with sample size (number of males and number of sounds per male) but remained high even when considering few calls per male in large groups. In accordance with the observed C.V.b:C.V.w ratios, the most important variables to allow male identification were $\mathrm{P}_{2}$ dominant frequency followed by dominant frequency modulation (the ratio between $\mathrm{P}_{1}$ and $\mathrm{P}_{2}$ dominant frequencies). $\mathrm{P}_{2}$ pulse period, amplitude modulation and total boatwhistle duration were also consistently important to discriminate among individuals in the various DFAs.

In the field, males can probably only detect a maximum of eight males at a time (maximum size of a chorus; unpubl. data) and call often in duets or in trios, thus potentially making the task of individual recognition simpler than the 13 males considered in the present study. Moreover, because males call often at rates of $c .10$ boatwhistles $\min ^{-1}$ (pers. obs.) they will easily experience more than the 16 calls from a neighbour having more opportunity to access distinct features from stationary nesting conspecifics.

In order for the above five features to be good candidates for individual identification, they should propagate well through the environment and should also be recognized by the central nervous system of the receiver. Sound propagation in shallow water can result in signal degradation over short distances, including sound pressure level and frequency attenuation, and temporal patterning loss (Mann, 2006; also see Fig. 3). Boatwhistles are thought to function both to announce territorial ownership and position to other males and to attract females as prospective mates (Winn, 1967). Because males can nest $<0.5 \mathrm{~m}$ apart (pers. obs.) environmental degradation of call properties should not impose a major restriction between male neighbours. The effect of attenuation and signal degradation, however, should be important for female attraction. This problem has probably been overcome by the increased acoustic output resulting from $H$. didactylus male choruses. If there is mate choice based on acoustic signals, it probably takes place when females are already in close range to males with access to minimally degraded signals. 
Differences among males in frequency attributes should be perceived by the $H$. didactylus although batrachoidids are hearing generalists, i.e. they lack morphological specializations that enhance the detection of the sound pressure component of the acoustic signals (Fay \& Simmons, 1999). According to Vasconcelos et al. (2007), dominant and fundamental frequencies of boatwhistles match the best hearing range of the species. $\mathrm{P}_{2}$ dominant frequency differs among individuals between $>10$ and $100 \%$ (Fig. 5), thus falling within the range of frequency discrimination ability of hearing generalists, which is generally slightly $>10 \%$ difference (Fay \& Simmons, 1999). Differences in frequency modulation should also be detected because disparities between $\mathrm{P}_{1}$ and $\mathrm{P}_{2}$ dominant frequencies are in the majority of the studied males $>10 \%$ (Fig. 5). This variable shows high interindividual variability and dominant frequency can be modulated upward or downward (Table I and Fig. 5). The large differences in signal duration found in the present study (but not pulse period) should also fall into the hearing discrimination abilities of $H$. didactylus since other batrachoidids can detect small differences in signal duration (McKibben \& Bass, 1998).

Acoustic recognition systems have arisen in situations where crowding, noisy backgrounds (such as in dense colonies of birds) or darkness reduce the roles of olfactory and visual cues or increase the risk of confusion (Beecher, 1989; Sayigh et al., 1999). Likewise, acoustic recognition is also beneficial when vocal animals defend long-term territories. In this context, individual recognition is adaptive because animals can direct less aggression to familiar neighbours, which are less likely to intrude into their territories. This phenomenon, known as the 'dear enemy effect' (Fischer, 1954), has been described in several animals (Temeles, 1994). In fishes, acoustic recognition has only been demonstrated in a coral reef species that breed in dense colonies. Myrberg \& Riggio (1985) tested the 'dear enemy effect' with the bicolour damselfish Stegastes partitus (Poey) and verified that males can recognize territorial neighbours based on acoustic cues, probably the dominant frequency that decreased pronouncedly with male size. Likewise, $H$. didactylus males establish long-term territories forming dense breeding aggregations. In addition, they live in turbid environments where vision is impaired. Consequently, being able to discriminate among different individuals would be beneficial in this species. A comparable social system where individual recognition has been demonstrated is found in anurans. Frogs and toads also form breeding choruses and establish long-term territories during the reproductive season and may show vocal individual recognition. For example, male bullfrogs Rana catesbeiana, Shaw can learn about individually distinct acoustic features of neighbours' calls and a neighbour's position by repeatedly hearing the call from a particular location (Bee \& Gerhardt, 2001).

This study was based on short periods of recordings from unseen fish. Although the identity of the sound producers cannot be completely ascertained, the present results suggest that there is enough information in the mating calls of the $H$. didactylus to promote individual recognition. Future work carried out with fully identified males will need to address whether boatwhistle characteristics are constant over longer periods of time and whether they are related to male features. 
Special thanks are given to F. Almada and J. Marques for helping with recordings, to P. Fonseca and J. M. Simões for their help with the sound playbacks and comments on the manuscript. This study was supported by the pluriannual programme (UI\&D 331/94) / FCT, and the grants POSI SFRH/BPD/14570/2003 (MCPA) and SFRH/ $\mathrm{BD} / 30491 / 2006$ (ROV) of FCT.

\section{References}

Amorim, M. C. P. (2006). Diversity of sound production in fish. In Communication in Fishes, Vol. I (Ladich, F., Collin, S. P., Moller, P. \& Kapoor, B. G., eds), pp. 71104. Enfield: Science Publishers.

Amorim, M. C. P., Vasconcelos, R. O., Marques, J. F. \& Almada, F. (2006). Seasonal variation of sound production in the Lusitanian toadfish, Halobatrachus didactylus. Journal of Fish Biology 69, 1892-1899. doi: 10.1111/j.1095-8649.2006.01247.x

Amorim, M. C. P., Simões, J. M. \& Fonseca, P. J. (2008). Acoustic communication in the Lusitanian toadfish. Halobatrachus didactylus: evidence for an unusual large vocal repertoire. Journal of Marine Biological Association of the United Kingdom 88, 1069 1073.

Barimo, J. F. \& Fine, M. L. (1998). Relationship of swim-bladder shape to the directionality pattern of underwater sound in the oyster toadfish. Canadian Journal of Zoology 76, 134-143.

Bee, M. A. \& Gerhardt, H. C. (2001). Neighbour-stranger discrimination by territorial male bullfrogs (Rana catesbeiana): II. Perceptual basis. Animal Behaviour 62, 11411150.

Bee, M. A., Kovich, C. E., Blackwell, K. J. \& Gerhardt, H. C. (2001). Individual variation in advertisement calls of territorial male green frogs, Rana clamitans: implications for individual discrimination. Ethology 107, 65-84. doi: 10.1046/ j.1439-0310.2001.00640.x

Beecher, M. D. (1989). Signalling systems for individual recognition: an information theory approach. Animal Behaviour 38, 248-261.

Bradbury, J. W. \& Vehrencamp, S. L. (1998). Principles of Animal Communication. Sunderland: Sinauer Associates.

Brantley, R. K. \& Bass, A. H. (1994). Alternative male spawning tactics and acoustic signals in the plainfin midshipman fish, Porichthys notatus (Teleostei, Batrachoididae). Ethology 96, 213-232.

Christie, P. J., Mennill, D. J. \& Ratcliffe, L. M. (2004). Chickadee song structure is individually distinctive over long broadcast distances. Behaviour 141, 101-124.

Crawford, J. D., Cook, A. P. \& Heberlein, A. S. (1997). Bioacoustic behavior of African fishes (Mormyridae): potential cues for species and individual recognition in Pollimyrus. The Journal of the Acoustical Society of America 102, 1200-1212.

Edds-Walton, P. L., Mangiamele, L. A. \& Rome, L. C. (2002). Variation of pulse repetition rate in boatwhistle sounds from oyster toadfish Opsanus tau around Waquoit bay, Massachusetts. Bioacoustics 13, 153-173.

Fay, R. R. \& Simmons, A. M. (1999). The sense of hearing in fishes and amphibians. In Comparative Hearing: Fish and Amphibians (Fay, R. R. \& Popper, A. N., eds), pp. 269-318. New York: Springer.

Fine, M. L. (1978). Seasonal and geographical variation of the mating call of the oyster toadfish Opsanus tau L. Oecologia 36, 45-57.

Fine, M. L. \& Lenhardt, M. L. (1983). Shallow-water propagation of the toadfish mating call. Comparative Biochemistry and Physiology A 76, 225-231.

Fine, M. L. \& Thorson, R. F. (2008). Effects of passive acoustics for assessing behavioral interactions in individual toadfish. Transactions of the American Fisheries Society 137, 627-637.

Fine, M. L., Malloy, K. L., King, C., Mitchell, S. L. \& Cameron, T. M. (2001). Movement and sound generation by the toadfish swimbladder. Journal of Comparative Physiology A 187, 371-379. 
Fischer, J. B. (1954). Evolution and bird sociality. In Evolution as a Process (Huxley, J., Hardy, A. C. \& Ford, E. B., eds), pp. 71-83. London: Allen and Unwin.

Ibara, R. M., Penny, L. T., Ebeling, A. W., van Dykhuizen, G. \& Caillet, G. (1983). The mating call of the plainfin midshipman, Porichthys notatus. In Predators and Prey (Noakes, D. G. L., Lindquist, D. G., Helfman, G. S. \& Ward, J. A., eds), pp. 205212. The Hague: Junk Press.

Jouventin, P., Aubin, T. \& Lengagne, T. (1999). Finding a parent in a king penguin colony: the acoustic system of individual recognition. Animal Behaviour 57, 11751183.

Knapp, R., Wingfield, J. C. \& Bass, A. H. (1999). Steroid hormones and paternal care in the plainfin midshipman fish (Porichthys notatus). Hormones and Behavior 35, 81-89.

Lamml, M. \& Kramer, B. (2006). Differentiation of courtship songs in parapatric sibling species of dwarf stonebashers from southern Africa (Mormyridae, Teleostei). Behaviour 143, 783-810.

Mann, D. A. (2006). Propagation of fish sounds. In Communication in Fishes, Vol. I (Ladich, F., Collin, S. P., Moller, P. \& Kapoor, B. G., eds), pp. 107-120. Enfield: Science Publishers.

McKibben, J. R. \& Bass, A. H. (1998). Behavioral assessment of acoustic parameters relevant to signal recognition and preference in a vocal fish. The Journal of the Acoustical Society of America 104, 3520-3533.

Modesto, T. \& Canário, A. V. M. (2003). Morphometric changes and sex steroid levels during the annual reproductive cycle of the Lusitanian toadfish, Halobatrachus didactylus. General and Comparative Endocrinology 131, 220-231.

Myrberg, A. A. Jr \& Riggio, R. J. (1985). Acoustically mediated individual recognition by a coral reef fish (Pomacentrus partitus). Animal Behaviour 33, 411-416.

Myrberg, A. A. Jr, Ha, S. J. \& Shamblott, H. (1993). The sounds of bicolor damselfish (Pomacentrus partitus): predictors of body size and a spectral basis for individual recognition and assessment. The Journal of the Acoustical Society of America 94, 3067-3070.

Remage-Healy, L. \& Bass, A. H. (2005). Rapid elevations in both steroid hormones and vocal signalling during playback challenge: a field experiment in gulf toadfish. Hormones and Behavior 47, 297-305.

Roux, C. (1986). Batrachoididae. In Fishes of the North-eastern Atlantic and the Mediterranean, Vol. III (Whitehead, P. J. P., Bauchot, M.-L., Hureau, J. C., Nielsen, J. \& Tortonese, E., eds), pp. 1360-1361. Paris: Unesco.

dos Santos, M., Modesto, T., Matos, R. J., Grober, M. S., Oliveira, R. F. \& Canario, A. (2000). Sound production by the Lusitanian toadfish, Halobatrachus didactylus. Bioacoustics 10, 309-321.

Sayigh, L. S., Tyack, P. L., Wells, R. S., Solows, A. R., Scott, M. D. \& Irvine, A. B. (1999). Individual recognition in wild bottlenose dolphins: a field test using playback experiments. Animal Behaviour 57, 41-50.

Skoglund, C. R. (1961). Functional analysis of swimbladder muscles engaged in sound production in the toadfish. Journal of Biophysical and Biochemical Cytology 10, 187-200.

Speirs, A. H. \& Davis, L. S. (1991). Discrimination by the Adélie penguins, Pygoscelis adeliae, between the loud mutual calls of mates, neighbours and strangers. Animal Behaviour 41, 937-944.

Temeles, E. J. (1994). The role of neighbours in territorial systems: when are they 'dear enemies'? Animal Behaviour 47, 339-350.

Thorson, R. F. \& Fine, M. L. (2002a). Crepuscular changes in emission rate and parameters of the boatwhistle advertisement call of the gulf toadfish, Opsanus beta. Environmental Biology of Fishes 63, 321-331.

Thorson, R. F. \& Fine, M. L. (2002b). Acoustic competition in the gulf toadfish Opsanus beta: acoustic tagging. The Journal of the Acoustical Society of America 111, 2302 2307. 
Vasconcelos, R. O., Amorim, M. C. P. \& Ladich, F. (2007). Effects of ship noise on the auditory sensitivity and acoustic communication in the Lusitanian toadfish. The Journal of Experimental Biology 210, 2104-2112.

Winn, H. E. (1967). Vocal facilitation and the biological significance of toadfish sounds. In Marine Bio-Acoustics, Vol. 2 (Tavolga, W. N., ed.), pp. 283-304. Oxford: Pergamon Press. 